\title{
COMPARAÇÃO ENTRE OS MÉTODOS DIRETO E INDIRETO DE DETERMINAÇÃO DO VO 2MÁX DE PRATICANTES DE CORRIDA
}

\author{
COMPARISON BETWEEN THE DIRECT AND INDIRECT METHODS OF VO ${ }_{2 M A ́ X}$ \\ DETERMINATION IN RUNNERS
}

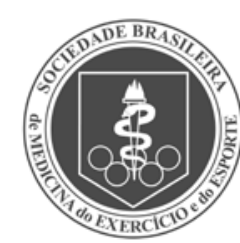

Artigo Original

\section{COMPARACIÓN ENTRE LOS MÉTODOS DIRECTO E INDIRECTO DE DETERMINACIÓN DEL VO ${ }_{2 M A X}$ DE PRACTICANTES DE CARRERA}

Ana Claudia Pelissari Kravchychyn ${ }^{1}$ (Nutricionista e Educadora Física) Julio César Camargo Alves ${ }^{1}$ (Educador Físico)

Terezinha Pelissari Kravchychyn' (Educadora Física)

Geraldo Ângelo Nogueira' (Médico)

Fabiana Andrade Machado (Educadora Física)

1. Universidade Estadual de Maringá. Maringá, PR, Brasil.

\section{Correspondência:}

Fabiana Andrade Machado Depto. de Educação Física - Bloco M 06, Sala 6. Campus Universitário. Universidade Estadual de Maringá (UEM).

Av. Colombo, 5.790.

87020-900. Maringá. PR. Brasil. famachado_uem@hotmail.com

\section{RESUMO}

Introdução: testes diretos são considerados "padrão ouro" para determinar variáveis fisiológicas, porém o seu custo financeiro é elevado e há a necessidade de mão de obra especializada para sua operacionalização. Sendo assim, os testes indiretos são utilizados pela maioria dos profissionais de Educação Física como uma alternativa acessível à falta da medida direta. Objetivo: comparar os valores de consumo máximo de oxigênio $\left(\mathrm{VO}_{2 \text { máx }}\right)$ determinados diretamente por um sistema de espirometria com valores determinados a partir de protocolos indiretos. Métodos: participaram 15 pessoas do gênero masculino (27,4 $\pm 3,5$ anos), fisicamente ativas, que realizaram o teste incremental em esteira rolante para determinação direta do $\mathrm{VO}_{2 \text { máx }}$ com analisador de gases. Os testes para predição do $\mathrm{VO}_{2 \text { máx }}$ foram: ErgoPC; Polar Fitness Test; testes do banco e de Cooper. Foi utilizada a estatística descritiva (média \pm desvio padrão - DP); a esfericidade foi testada pelo teste de Mauchly, os métodos foram comparados por ANOVA de medidas repetidas com o ajuste de Bonferroni para comparações múltiplas. A normalidade dos dados foi aferida pelo teste de Shapiro-Wilk e também foi aplicado o teste coeficiente de correlação de Pearson, adotando-se $p<0,05$. Resultados: o valor médio de $\mathrm{VO}_{2 \text { máx }}$ direto foi de $55,8 \pm 6,1 \mathrm{~m} / \mathrm{kg}^{-1} \cdot \mathrm{min}^{-1}$ e os valores para os testes indiretos, percentual de subestimação e coeficiente de correlação, foram, respectivamente: ErgoPc 33,7 $\pm 4,5 \mathrm{ml} \cdot \mathrm{kg}^{-1} \cdot \mathrm{min}^{-1}(39,6 \%$; $r=0,71 ; p<0,001)$; Polar Fitness Test 53,2 $\pm 6,4 \mathrm{ml} \cdot \mathrm{kg}^{-1} \cdot \mathrm{min}^{-1}(4,6 \% ; r=0,64 ; p=0,774)$; teste do banco 48,8 $\pm 6,3 \mathrm{ml} \cdot \mathrm{kg}^{-1} \cdot \mathrm{min}^{-1}(12,5 \% ; r=0,60 ; P=0,001)$ e de Cooper $43,9 \pm 7,9 \mathrm{ml} \cdot \mathrm{kg}^{-1} \cdot \mathrm{min}^{-1}(21,4 \% ; r=0,65 ; p<0,001)$. Conclusão: os métodos indiretos podem sugerir sobrecarga de treino inferior ou superior para adaptações fisiológicas pretendidas por subestimarem o direto, sendo o Polar Fitness Test o mais indicado para uso diário, pois apresentou valores mais próximos ao valor direto.

Palavras-chave: protocolos, masculino, espirometria.

\section{ABSTRACT}

Introduction: direct tests are considered "gold standard" for determining physiological variables. However, the financial cost is high and there is the need of skilled labor for its operation. Thus, the indirect tests are used by most professional of Physical Education as an affordable alternative to the lack of direct measurement. Objective: to compare the values of maximum consumption of oxygen $\left(\mathrm{VO}_{2 \text { max }}\right)$, determined directly by a system with spirometry values determined from indirect protocols. Methods: 15 male individuals participated (27.4 \pm 3.5 years), physically active, who performed the incremental test on treadmill for the direct determination of $\mathrm{VO}_{2 \max }$ with the gas analyzer. The tests for the prediction of $\mathrm{VO}_{2 \max }$ were ErgoPC; Polar Fitness Test, test bank and Cooper. We used descriptive statistics (mean \pm standard deviation - SD); the sphericity was tested by Mauchly's test, the methods were compared by ANOVA of repeated measurements, with Bonferroni adjustment for multiple comparisons. Data normality was evaluated by the Shapiro-Wilk test, and the Pearson correlation coefficient was also applied, adopting a significance level of $p<0.05$. Results: the average $\mathrm{VO}_{2 \max }$ was determined directly from $55.8 \pm 6.1 \mathrm{~m} / \cdot \mathrm{kg}^{-1} \cdot \mathrm{min}^{-1}$ and the values for the indirect tests, the percentage of underestimation and correlation coefficient were, respectively: 33.7 ErgoPC \pm $4.5 \mathrm{~m} / \cdot \mathrm{kg}^{-1} \cdot \mathrm{min}^{-1}(39.6 \%, r=0.71, p<0.001)$; Polar Fitness Test $53.2 \pm 6.4 \mathrm{~m} / \cdot \mathrm{kg}^{-1} \cdot \mathrm{min}^{-1}(4.6 \%, r=0.64, p=0.774)$; bank test $48.8 \pm 6.3 \mathrm{ml} \cdot \mathrm{kg}^{-1} \cdot \mathrm{min}^{-1}(12.5 \%, r=0.60, p=0.001)$ and Cooper $43.9 \pm 7.9 \mathrm{ml} \cdot \mathrm{kg}^{-1} \cdot \mathrm{min}^{-1}(21.4 \%, r$ $=0.65, p<0.001)$. Conclusion: the indirect methods may suggest overload in lower or higher training for required physiological adaptations and only the Polar Fitness Test showed no difference statistically significant.

Keywords: protocols, male, spirometry.

\section{RESUMEN}

Introducción: los tests directos son considerados "estándar oro" para determinar variables fisiológicas, sin embargo su costo financiero es elevado y se necesita mano de obra especializada para su operacionalización. siendo así, los tests indirectos son usados por la mayoría de los profesionales de Educación Física como una 
alternativa accesible a la falta de la medida directa. Objetivo: comparar los valores de consumo máximo de oxígeno $\left(\mathrm{VO}_{2 \text { máx }}\right)$ determinados directamente por un sistema de espirometría con valores determinados a partir de protocolos indirectos. Métodos: participaron 15 sujetos del género masculino (27,4 $\pm 3,5$ años), físicamente activos que realizaron el test incremental en cinta para determinación directa del $\mathrm{VO}_{2 m a ́ x}$ Con analizador de gases. Los tests para predicción del $\mathrm{VO}_{2 \text { máx }}$ fueron: ErgoPC; Polar Fitness Test; tests del banco y de Cooper. Fue utilizada la estadística descriptiva (promedio \pm desvío estándar - DP); la esfericidad fue probada por el test de Mauchly, los métodos fueron comparados por ANOVA de medidas repetidas con el ajuste de Bonferroni para comparaciones múltiples. La normalidad de los datos fue probada a través del test de Shapiro-Wilky aplicado el test coeficiente de correlación de Pearson, adoptándose $p<0,05$. Resultados: el valor promedio de $\mathrm{VO}_{2 \text { máx }}$ directo fue de $55,8 \pm 6,1 \mathrm{~m} / \mathrm{kg}^{-1} \cdot \mathrm{min}^{-1}$ y $\mathrm{los}$ valores para los tests indirectos, porcentual de subestimación y coeficiente de correlación fueron, respectivamente: ErgoPC 33,7 $\pm 4,5 \mathrm{ml} \cdot \mathrm{kg}^{-1} \cdot \mathrm{min}^{-1}(39,6 \% ; r=0,71 ; p<0,001)$; Polar Fitness Test 53,2 $\pm 6,4 \mathrm{ml} \cdot \mathrm{kg}^{-1} \cdot \mathrm{min}^{-1}(4,6 \% ; r=0,64 ;$ $p=0,774)$; test del banco 48,8 $\pm 6,3 \mathrm{ml} \cdot \mathrm{kg}^{-1} \cdot \mathrm{min}^{-1}(12,5 \% ; r=0,60 ; P=0,001)$ y de Cooper $43,9 \pm 7,9 \mathrm{ml} \cdot \mathrm{kg}^{-1} \cdot \mathrm{min}^{-1}$ $(21,4 \% ; r=0,65 ; p<0,001)$. Conclusión: los métodos indirectos pueden sugerir sobrecarga de entrenamiento inferior o superior para adaptaciones fisiológicas pretendidas, por subestimar el directo, siendo el Polar Fitness Test el más indicado para uso diario, pues presentó valores más próximos al valor directo.

Palabras-clave: protocolos, masculino, espirometría.

\section{INTRODUÇÃO}

O consumo máximo de oxigênio $\left(\mathrm{VO}_{2 \text { máx }}\right)$ é definido como a mais alta taxa de oxigênio consumida para realizar um esforço máximo' e sua determinação gera importantes informações acerca da capacidade de fornecimento de energia e nível de aptidão física dos sujeitos ${ }^{2,3}$. A determinação desta variável reflete a integração entre os sistemas respiratório, cardiovascular e neuromuscular fazendo com que o $\mathrm{VO}_{2 \text { máx }}$ mostre-se uma medida fundamental para a prescrição de treinamento ${ }^{4}$.

A medida direta do $\mathrm{VO}_{2 \text { máx }}$ realizada através de um sistema de espirometria analisa as frações expiradas de oxigênio $\left(\mathrm{O}_{2}\right)$ e dióxido de carbono $\left(\mathrm{CO}_{2}\right)$ durante o esforço e a ventilação pulmonar, sendo considerada "padrão ouro" por sua fidedignidade, possuindo custo elevado e exigindo mão de obra especializada para sua operacionalização, tornando-se de difícil acesso em muitas situações ${ }^{5,6}$.

Como alternativa para as adversidades encontradas para a utilização do método direto, foram desenvolvidos diversos protocolos de caráter indireto para determinação do $\mathrm{VO}_{2 \text { máx }}{ }^{7-10}$. Tais métodos determinam esta variável através de equações baseadas em tempo ou distância pré-estabelecidos, frequência cardíaca, idade, gênero, nível de condicionamento físico dentre outros fatores ${ }^{11}$. No entanto, estudos mostram que os protocolos indiretos podem subestimar ou superestimar o valor real do $\mathrm{VO}_{2 \text { máx }}$ comprometendo a forma como os dados podem ser utilizados ${ }^{6,12-14}$.

Poucos estudos compararam diferentes protocolos de determinação do $\mathrm{VO}_{2 \text { máx }}$ e até onde temos conhecimento não foram comparados protocolos indiretos acessíveis à profissionais da área de Educação Física, esportes e saúde a fim de auxiliar no menor erro de prescrição de cargas de treinamento e/ou na medida clínica de capacidade funcional do sistema cardiorrespiratório ${ }^{12}$.

Portanto, o objetivo do presente estudo foi comparar os valores de $\mathrm{VO}_{2 \text { máx }}$ determinados diretamente por um sistema de espirometria com valores determinados a partir de protocolos indiretos.

\section{MÉTODOS}

A amostra foi composta por 15 sujeitos do gênero masculino (27,4 $\pm 3,5$ anos de idade; massa corporal 78,1 $\pm 6,9 \mathrm{~kg}$; estatura 175,2 $\pm 5,3 \mathrm{~cm} ; \mathrm{IMC} 25,3 \pm 2,3 \mathrm{~kg} \cdot \mathrm{m}^{-2} ; 15,0 \pm 6,5 \% \mathrm{G}$ ) praticantes regulares de corrida (tempo médio de prática de cinco anos), com frequência de treinamento de três a cinco vezes por semana. A participação foi voluntária, isenta de qualquer recompensa e procedida após o preenchimento do termo de consentimento livre e esclarecido (TCLE). O projeto de pesquisa foi previamente aprovado pelo Comitê de Ética em pesquisa local (parecer \#719/2010).

Foi aplicada uma ficha de anamnese para a identificação do sujeito, verificação do estado de treinamento, integridade física e o estado saudável no início do experimento e avaliação antropométrica.

A determinação do $\mathrm{VO}_{2 \text { máx }}$ foi realizada por dois métodos: direto (espirometria) e indireto (fórmulas preditivas).

Para o método direto foi aplicado um protocolo específico com a realização de um teste de esforço máximo em ambiente laboratorial, utilizando um sistema de espirometria de circuito aberto que fornece informações respiratórias a cada 15 segundos (Fitmate, Cosmed ${ }^{\circledR}$ - Itália). O teste foi realizado em esteira elétrica multiprogramável (INBRAMED ATL, Porto Alegre, Brasil) e temperatura ambiente foi mantida entre $20^{\circ} \mathrm{C}$ e $24^{\circ} \mathrm{C}$. O protocolo incremental foi composto de um aquecimento de três minutos a $7 \mathrm{~km} \cdot \mathrm{h}^{-1}$, incrementos de $1 \mathrm{~km} \cdot \mathrm{h}^{-1}$ a cada três minutos e velocidade inicial de $9 \mathrm{~km} \cdot \mathrm{h}^{-1}$. Durante todo o teste foi mantida uma inclinação constante de $1 \%$ e os sujeitos foram encorajados verbalmente a permanecerem no teste o maior tempo possivel até exaustão voluntária. Todos os procedimentos foram acompanhados por um médico cardiologista.

Para a determinação indireta do $\mathrm{VO}_{2 \text { máx }}$ foram utilizadas fórmulas preditivas a partir da realização de protocolos específicos a cada uma delas (ErgoPC, teste de Cooper, teste do Banco e Polar Fitness Test).

O programa ErgoPC (Micromed, Brasília, Brasil) realiza os registros do comportamento cardíaco durante o teste de esforço predizendo, de forma indireta, $\mathrm{OVO}_{2 \text { máx }}$ através da fórmula proposta por Foster et al. ${ }^{15}$, que considera o aumento da intensidade em cada um dos estágios para determinar esta variável:

$\mathrm{VO}_{2 \text { máx }}\left(\mathrm{ml} \cdot \mathrm{kg}^{-1} \cdot \mathrm{min}^{-1}\right)=[0,869] \times\left[0,2 \times\right.$ Velocidade $\left(\mathrm{m} \cdot \mathrm{min}^{-1}\right)$

$+0,9 \times$ Velocidade $\times$ Elevação $(\% / 100)+3,5]+[-0,070]$

Foi aplicado o teste de andar e/ou correr por 12 minutos, sem interrupção, amplamente conhecido com teste de Cooper ${ }^{16}$. Este protocolo foi realizado em uma pista oficial de atletismo (400 m), sendo registrada, ao final de 12 minutos, a distância total percorrida em metros.

O teste do banco foi realizado segundo o protocolo de Quens 
College $^{9}$, que consiste em realizar subidas e decidas em um banco com altura de $41 \mathrm{~cm}$ seguindo a cadência de uma subida a cada 2,5 segundos sendo 24 subidas por minuto. O teste teve duração de três minutos e a frequência cardíaca ( $F C$ ) foi registrada do quinto até o $22^{\circ}$ minuto após o término do teste. $\mathrm{O} \mathrm{VO}_{2 \text { máx }}$ foi predito através da fórmula:

$$
\mathrm{VO}_{2 \text { máx }}\left(\mathrm{ml}^{\mathrm{kg}} \mathrm{kg}^{-1} \cdot \mathrm{min}^{-1}\right)=111,33-(0,42 \times \mathrm{FC})
$$

O protocolo do Polar Fitness Test baseia-se na FC de repouso $\left(F C_{\text {rep }}\right)$, idade, gênero, estatura, massa corporal e nível de atividade física auto-avaliada do sujeito. Os dados foram inseridos no sistema de um frequencimetro da marca Polar RS200 e em seguida o indivíduo permaneceu sentado durante cinco minutos para que o $\mathrm{VO}_{2 \text { máx }}$ fosse estimado a partir dos parâmetros acima citados.

\section{Análise Estatística}

Todas as análises foram realizadas pelo programa SPSS (Statistical Package for the Social Sciences - SPSS). Foi utilizada estatística descritiva (media \pm desvio padrão - DP), a normalidade dos dados foi testada pelo teste de Shapiro-Wilk; a comparação entre os métodos direto e indireto foi realizada a partir da Analysis of Variance (ANOVA) de medidas repetidas. Foi realizado o teste de Mauchly para verificar a esfericidade. Caso esse pressuposto não fosse assumido, foi aplicado o ajuste de Greenhouse-Geisser. Havendo diferenças na ANOVA, foi empregado o ajuste de Bonferroni para as comparações múltiplas. Foi utilizado o coeficiente de correlação de Pearson para verificar a relação entre os métodos. Por fim, foram apresentados os plots de Bland e Altman para a análise de concordância entre os métodos, adotando-se nível de significância de $p<0,05$.

O objetivo do presente estudo foi comparar os valores de $\mathrm{VO}_{2 \text { máx }}$ determinados diretamente por um sistema de espirometria com valores determinados a partir de protocolos indiretos.

\section{RESULTADOS}

Na tabela 1 estão apresentados os valores médios \pm desvio padrão (DP) de $\mathrm{VO}_{2 \text { máx }}\left(\mathrm{ml} \cdot \mathrm{kg}^{-1} \cdot \mathrm{min}^{-1}\right)$ obtidos através dos métodos direto (espirometria) e indiretos (ErgoPC, teste de Cooper, teste do Banco e Polar ${ }^{\circledR}$ Fitness Test). Houve diferença estatisticamente significante entre os valores de $\mathrm{VO}_{2 \text { máx }}$ determinado diretamente pelo sistema de espirometria comparado aos valores preditos pelos protocolos ErgoPC, teste de Cooper e teste do Banco. O valor do $\mathrm{VO}_{2 \text { máx }}$ predito pelo Polar Fitness Test não apresentou diferença estatisticamente significante em relação ao valor determinado diretamente pelo sistema de espirometria.

Para todos os protocolos de determinação do $V_{2} O_{2 \text { máx }}$ foi encontrada correlação do método direto com os valores indiretamente preditos: ErgoPC $r=0,71$; Teste de Cooper $r=0,65$; Teste do Banco $r=0,60$ e Polar Fitness Test $r=0,64$.

A figura 1 mostra os plots da análise de concordância do método

Tabela 1. Valores médios $\pm \mathrm{DP}$ do $\mathrm{VO}_{2 \text { máx }}$ determinado pelos diferentes métodos e protocolos e diferenças absolutas e relativas (\%) em relação ao método direto (espirometria) $(n=15)$

\begin{tabular}{|c|c|c|c|}
\hline Métodos & $\begin{array}{c}\mathrm{VO}_{2 \text { máx }} \\
\left(\mathrm{ml} \cdot \mathrm{kg}^{-1} \cdot \mathrm{min}^{-1}\right)\end{array}$ & $\begin{array}{c}\text { Diferença absoluta } \\
\left(\mathrm{ml} \cdot \mathrm{kg}^{-1} \cdot \mathrm{min}^{-1}\right)\end{array}$ & $\begin{array}{c}\text { Diferença relativa } \\
(\%)\end{array}$ \\
\hline Espirometria & $55,8 \pm 6,1$ & - & - \\
\hline ErgoPC & $33,7 \pm 4,5^{*}$ & $-22,1 \pm 4,3$ & $-39,5 \pm 5,9$ \\
\hline Cooper & $43,9 \pm 7,0^{*}$ & $-11,9 \pm 6,1$ & $-21,5 \pm 11,2$ \\
\hline Banco & $48,8 \pm 6,2^{*}$ & $-7,0 \pm 5,5$ & $-12,2 \pm 9,8$ \\
\hline Polar & $53,2 \pm 6,3$ & $-2,6 \pm 5,3$ & $-4,3 \pm 9,3$ \\
\hline
\end{tabular}
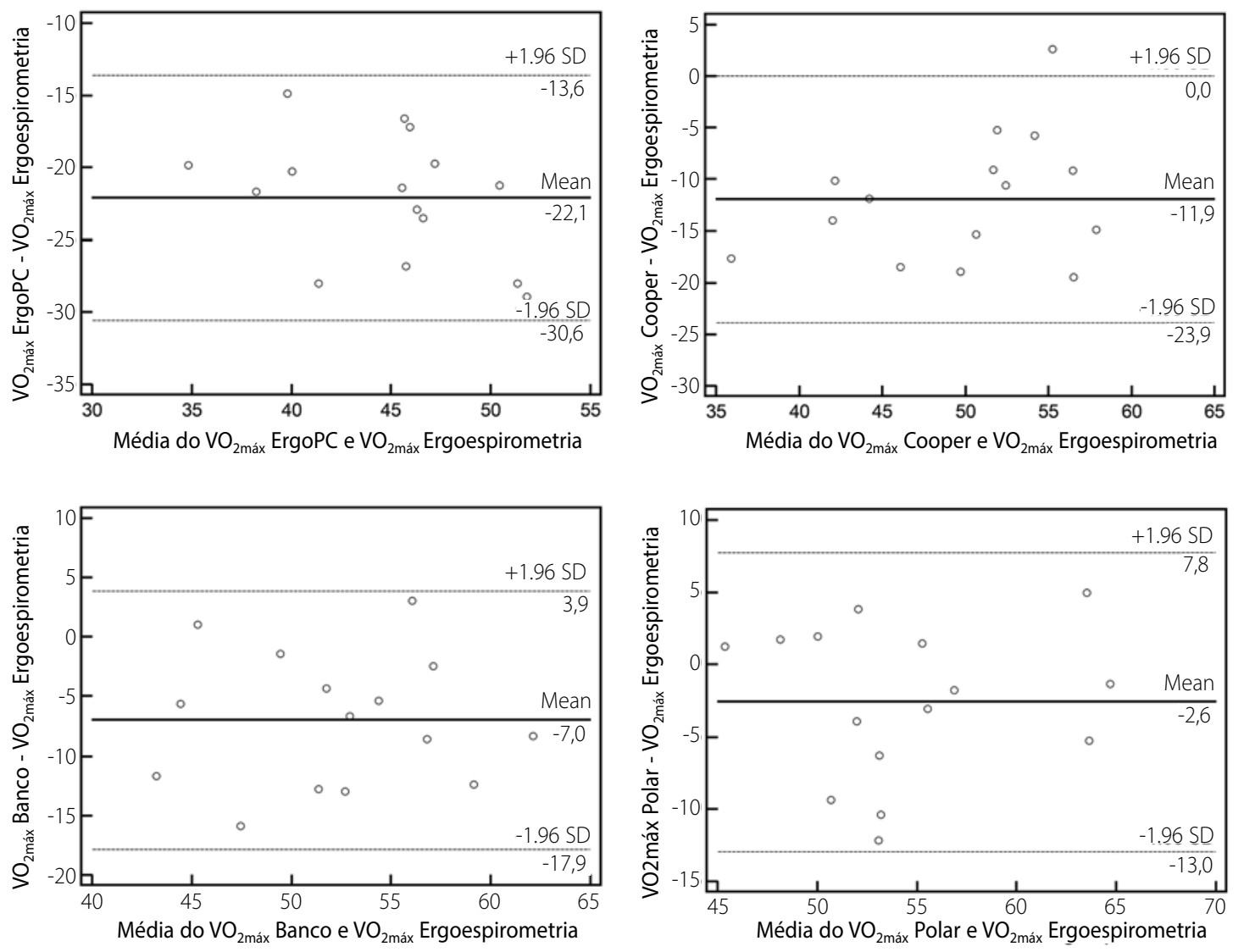

Figura 1. Análise de concordância de Bland e Altman para os métodos direto e indireto. 
direto com relação aos métodos indiretos, permitindo visualizar as diferenças médias e os limites extremos de concordância apresentada pelas determinações dos métodos. Os limites de concordância mostram que houve boa concordância de todos os métodos indiretos em relação ao método direto. No entanto, as diferenças médias entre o valor de $\mathrm{VO}_{2 \text { máx }}$ obtido a partir do método direto e os valores obtidos pelos métodos indiretos, expressos em valores relativos $\left(\mathrm{ml} \cdot \mathrm{kg}^{-1} \cdot \mathrm{min}^{-1}\right)$ foram: ErgoPC -22,1; Teste de Cooper -11,9; Teste do Banco -7,0 e Polar Fitness Test -2,6.

\section{DISCUSSÃO}

Como principal achado do estudo destaca-se que o valor de $\mathrm{VO}_{2 \text { máx }}$ determinado pelo método Polar ${ }^{\circledR}$ Fitness Test quando comparado com o método direto não apresentou diferença estatisticamente significante. Apesar das demais comparações dos valores de $\mathrm{VO}_{2 \text { máx }}$ determinados por protocolos indiretos subestimarem o valor direto, as correlações encontradas classificamse como fortes, incluindo a medida estimada pelo Polar Fitness Test.

$\mathrm{O}$ valor do $\mathrm{VO}_{2 \text { máx }}$ determinado pelo protocolo ErgoPC corroborou os dados encontrados por Peserico et al. ${ }^{12}$ no qual foi comparado o mesmo método indireto com o $\mathrm{VO}_{2 \text { máx }}$ de mulheres corredoras determinado através da espirometria de circuito aberto (Espirômetro V02000 Inbrasport, Porto Alegre Brasil), encontrando uma subestimação em relação aos valores determinados diretamente (Direto: $51,8 \pm 6,8 \mathrm{ml} \cdot \mathrm{kg}^{-1} \cdot \mathrm{min}^{-1}$; Indireto: $\left.42,8 \pm 3,7 \mathrm{ml} \cdot \mathrm{kg}^{-1} \cdot \mathrm{min}^{-1}\right)$. Alguns estudos mostram valores que superestimaram a medida direta, apresentando valores de ErgoPC contrários a este estudo ${ }^{17-21}$.

Como exemplos de valores superestimados pelo protocolo ErgoPC, Rondon et al. ${ }^{21}$ encontraram um valor do $\mathrm{VO}_{2 \text { máx }}$ de 53,0 \pm $7,5 \mathrm{ml} \cdot \mathrm{kg}^{-1} \cdot \mathrm{min}^{-1}$ (valor determinado indiretamente) e de 42,1 $\pm 3,5$ $\mathrm{ml} \cdot \mathrm{kg}^{-1} \cdot \mathrm{min}^{-1}$ (valor determinado diretamente). Santos et al. ${ }^{20}$ (2007) encontraram, para homens e mulheres, um valor indireto de 59,5 \pm $5,6 \mathrm{ml} \cdot \mathrm{kg}^{-1} \cdot \mathrm{min}^{-1}$ e $49,1 \pm 3,1 \mathrm{ml} \cdot \mathrm{kg}^{-1} \cdot \mathrm{min}^{-1}$, respectivamente e direto de $50,6 \pm 8,1 \mathrm{ml} \cdot \mathrm{kg}^{-1} \cdot \mathrm{min}^{-1}$ e $34,4 \pm 7,0 \mathrm{ml} \cdot \mathrm{kg}^{-1} \cdot \mathrm{min}^{-1}$, respectivamente. Estes resultados controversos entre os estudos pode ser explicado através da analise da especificidade dos testes cardiológicos feitos em clinicas médicas que, não possuem como intuito principal analisar $0 \mathrm{VO}_{2 \text { máx }}$ e sim de avaliar a condição cardíaca do sujeito em esforço, inserido a fórmula preditiva de $\mathrm{VO}_{2 \text { máx }}$ entre os seus resultados.

Os equipamentos de eletrocardiogramas (ECG), assim como o sistema ErgoPC, são utilizados na área clínica para determinação do $\mathrm{VO}_{2 \text { máx }}$ em protocolos de esforço máximo e podem trazer erros nas medidas da potência cardiorrespiratória do indivíduo. Sendo assim, este método assume uma relevância e necessidade de uma proximidade do valor direto, tendo em vista que os estudos apresentados não concordam entre si, sub ou superestimando a medida direta, e que uma parcela significativa dos testes de esforço é conduzida por um cardiologista.

O protocolo de Cooper é um dos métodos indiretos mais utilizados por profissionais da área de Esporte e Educação Física devida sua grande facilidade de aplicação, baixo custo e a possibilidade de várias pessoas serem avaliadas ao mesmo tempo 22; porém, também subestimou o método direto. Costa et al..$^{19}$ corrobora com os dados encontrados no presente estudo, pois conclui que o teste de Cooper subestimou as intensidades dos valores de 50\% e 85\% do $\mathrm{VO}_{2 \text { máx }}$ quando comparado com a medida direta.

O teste do banco é muito utilizado em academias por sua facilidade de aplicação, viabilidade de tempo e segurança devido a sua característica submáxima ${ }^{23,24}$; porém, o resultado encontrado para teste do banco subestimou o valor da medida direta do $\mathrm{VO}_{2 \text { máx }}$ como os outros protocolos (Direto: $55,8 \pm 6,1 \mathrm{ml} \cdot \mathrm{kg}^{-1} \cdot \mathrm{min}^{-1}$; Indireto: 48,8 \pm $\left.6,2 \mathrm{ml} \cdot \mathrm{kg}^{-1} \cdot \mathrm{min}^{-1}\right)$. Em contrapartida Chatterjee et al. ${ }^{25}$ compararam o $\mathrm{VO}_{2 \text { máx }}$ determinado diretamente com teste do banco em estudantes do gênero feminino, detectando que houve uma superestimação dos valores de $\mathrm{VO}_{2 \max }$ determinados indiretamente pelo teste do banco com os valores determinado diretamente, tornando conflitantes também os resultados para o protocolo do banco (Direto: 32,8 $\pm 3,8$ $\mathrm{ml} \cdot \mathrm{kg}^{-1} \cdot \mathrm{min}^{-1}$; Indireto: $35,5 \pm 4,4 \mathrm{ml} \cdot \mathrm{kg}^{-1} \cdot \mathrm{min}^{-1}$ ).

O método Polar ${ }^{\circledR}$ Fitness Test se caracteriza por ser capaz de predizer a potência aeróbia máxima de uma pessoa a partir do estado de repouso. ${ }^{13}$ Kruel et al. ${ }^{13}$ testaram a validade e a confiabilidade do $\mathrm{VO}_{2 \text { máx }}$ estimado pelo Polar ${ }^{\circledR}$ Fitness Test em relação ao $\mathrm{VO}_{2 \text { máx }}$ direto. Não corroborando com os resultados do presente estudo, os autores encontraram uma superestimação do valor predito para o valor direto, porém concluíram que o método apresenta confiabilidade apresar de não ser confirmada a validade (Direto: $36,5 \pm 6,1 \mathrm{ml} \cdot \mathrm{kg}^{-1} \cdot \mathrm{min}^{-1}$; Indireto: 45,4 \pm 12,6 $\mathrm{ml} \cdot \mathrm{kg}^{-1} \cdot \mathrm{min}^{-1}$ )

Apesar de haver uma escassez de estudos que testam o método Polar ${ }^{\circledR}$ Fitness Test, no presente estudo, este método indireto não foi estatisticamente diferente do direto, apresentando-se como um bom método para se mensurar o $\mathrm{VO}_{2 \text { máx }}$ na pratica diária.

As divergências encontradas na literatura em relação à comparação dos valores estimados de $\mathrm{VO}_{2 \text { máx }}$ pelo método indireto com o método direto, podem ser explicadas pelo fato de que em todos os métodos indiretos utiliza-se fórmulas matemáticas com valores de variáveis indiretamente relacionadas com o $\mathrm{VO}_{2 \text { máx }}$ como por exemplo: máxima distância percorrida e tempo fixo, máxima velocidade atingida em teste incremental em esteira, frequência cardíaca de repouso, frequência cardíaca máxima, nível de treinamento, idade, gênero, hereditariedade, estado clínico cardiovascular, peso corporal e outros fatores ${ }^{26,27}$.

Estas variáveis estimam para mais ou para menos o valor $\mathrm{VO}_{2 \text { máx }}$ e, por estarem expostas a muitos fatores externos acabam se tornando variáveis frágeis, comprometendo os valores finais da estimativa. Além disto, as fórmulas matemáticas podem também apresentar erros. Por estes possíveis erros apresentados, tanto nas variáveis de predição quanto nas fórmulas matemáticas, o valor de $\mathrm{VO}_{2 \text { máx }}$ estimado pelos métodos indiretos sub ou superestimam o valor determinado pelo método direto. O método indireto que utiliza o maior número das variáveis citadas para predizer o $\mathrm{VO}_{2 \text { máx }}$ é o Polar ${ }^{\circledR}$ Fitiness Test, justificando assim sua maior aproximação em relação aos valores encontrados pelo método direto.

Apesar de todos os testes subestimarem e, três dos quatro testes apresentarem diferença significativa para o valor do $\mathrm{VO}_{2 \text { máx }}$ determinado diretamente, todos os métodos se correlacionaram com o método direto (Espirometria), apontando que mesmo em condição de subestimação todos os protocolos podem ser utilizados por profissionais da área por apresentarem correlação com o protocolo direto, pontuando que todas as limitações devem ser consideradas. Outros estudos realizados com alguns destes protocolos também analisados, mostraram uma alta correlação dos métodos indiretos com o valor direto $10,12,25,28$.

Contudo, a utilização de métodos indiretos de determinação do $\mathrm{VO}_{2 \text { máx }}$ devem ser utilizados com critério e não devem ser o único parâmetro para prescrição de treinamento, podendo ser trabalhada em conjunto com outras variáveis fisiológicas, pois as intensidades advindas de protocolos indiretos podem atribuir uma sobrecarga inferior ou superior às pretendidas. 
A comparação dos valores de $\mathrm{VO}_{2 \text { máx }}$ determinados direta e indiretamente de forma transversal, pode caracterizar uma limitação do estudo, visto que, protocolos indiretos respondem de forma positiva às adaptações do treinamento em acompanhamentos longitudinais, facilitando assim a escolha de qual método utilizar neste tipo de prescrição, caso não haja a possibilidade da aplicação do método direto.

\section{CONCLUSÃO}

Os resultados do estudo mostram que os protocolos indiretos (Cooper, ErgoPC e teste do Banco de determinação do $\mathrm{VO}_{2 \text { máx }}$ ) subestimaram o valor direto. Sendo assim, estes protocolos não podem estar entre os mais recomendados para esse tipo de determinação por apresentarem limitações em relação a delimitação da capacidade aeróbia. Porém, a correlação observada com os valores de $\mathrm{VO}_{2 \text { máx }}$ determinado diretamente mostrou que desde de que o profissional leve em consideração populações semelhantes a amostra e o percentual de subestimação, os protocolos de Cooper, ErgoPC e teste do Banco podem ser utilizados na área clínica, esportes e Educação Física; sendo o método do Polar Fitness Test o que mais se aproxima da medida direta podendo ser utilizado em substituição a este método apresentando menor margem de erro.

Todos os autores declararam não haver qualquer potencial conflito de interesses referente a este artigo.

\section{REFERÊNCIAS}

1. Astrand PO. Experimental studies of physical working capacity in relation to sex and age. Copenhagen: Munksgaard; 1952.

2. Caputo F, Oliveira MFM, Greco CC, Denadai BS. Exercício aeróbio: Aspectos bioenergéticos, ajustes fisiológicos, fadiga e índices de desempenho. Rev Bras Cineantropom Desempenho Hum. 2009;11(1):94-102.

3. Basset JR, Howley ET. Limiting factors for maximum oxygen uptake and determinants of endurance performance. Med Sci Sports Exerc. 2000;32(1):70-84.

4. Atterhog JH, Jonsson B, Samuelsson R. Exercise testing: a prospective study of complication rates. Am Heart J 1979;98(5):572-9.

5. Santos $T M$, Viana BF, Sá Filho A. Reprodutibilidade do VO2máx estimado na corrida pela frequência cardíaca e consumo de oxigênio de reserva. Rev Bras Educ Fís Esporte. 2012;26(1):29-36.

6. Guimarães J, Stein R, Vilas-Boas F. Normatização de técnicas e equipamentos para realização de exames em ergometria e ergoespirometria. Arq Bras Cardiol. 2003;80:458-64.

7. Santos TM, Rodrigues Al, Greco CC, Marques AL, Terra BS, Oliveira BRR. VO2máx estimado e sua velocidade correspondente predizem o desempenho de corredores amadores. Rev Bras Cineantropom Desempenho Hum. 2012;14(2):192-201.

8. Sousa MS. Teste de banco: adequação da altura do ergômetro a estatura para indivíduos a partir de 09 anos de idade, de ambos os sexos praticantes e não praticantes de atividade física [dissertação]. Campinas: Universidade Estadual de Campinas (Unicamp); 1997.

9. Mcardle WD, Katch FI, Katch VL. Exercise physiology. Philadelphia: Lea and Fabiger; 1981.

10. Cooper KH. A means of assessing maximal oxygen intake. Correlation between field and treadmill testing. JAMA. 1968;203(3):201-4

11. American College of Sports Medicine. Guidelines for Exercise Testing and Prescription. 6nd. ed. Philadelphia: Lippincott, Williams \& Wilkins; 2000.

12. Peserico CS, Mezzaroba PV, Nogueira GA, Moraes SMF, Machado FA. Comparação entre os métodos direto e indireto de determinação do consumo máximo de oxigênio em mulheres corredoras. Rev Bras Med Esporte. 2011;17(4):270-3.

13. Kruel LFM, Coertjens M,Tartaruga LAP, Pusch HC. Validade e fidedignidade do consumo máximo de oxigênio predito pelo frequencímetro polar M52. Rev Bras Fisiol Exerc. 2003;2:147-56.

14. Mahseredjian F, Barros Neto TL, Tebexreni AS. Estudo comparativo de métodos para a predição do consumo máximo de oxigênio e limiar anaeróbio em atletas. Rev Bras Med Esporte. 1999;5(5):167-72.
15. Foster C, Crowe AJ, Daines E, Dumit M, Green MA, Lettau S, et al. Predicting functional capacity during treadmill testing independent of exercise protocol. Med Sci Sports Exerc. 1996;28(6):752-6.

16. Cooper KH. O programa aeróbico para o bem-estar total. Rio de Janeiro: Nórdica; 1982.

17. Filardo RD, Silva RCR, Petroski EL. Validação das equações metabólicas para caminhada e corrida propostas pelo American College of Sports Medicine em homens entre 20 e 30 anos de idade. Rev Bras Med Esporte. 2008;14(6):523-7

18. Brum PP, Sousa WAF, Santos MAA. Comparação entre as faixas de intensidade para exercício aeróbico propostas pelo ACSM com as obtidas na ergoespirometria. Rev Bras Ciênc Movimento. 2008;16(1):1-19.

19. Costa EC, Guerra LMM, Guerra FEF, Nunes N, Pontes Júnior FL. Validade da medida do consumo máximo de oxigênio e prescrição de intensidade de treinamento aeróbico preditos pelo teste de cooper de 12 minutos em jovens sedentários. RBPFEX. 2007;1(4):32-9.

20. Santos MAA. Análise da prescrição e da aplicabilidade do consumo de oxigênio de reserva durante o exercício aeróbio contínuo nas intensidades de $50 \%$ a $80 \%$ do consumo máximo de oxigênio [tese]. Espírito Santo: Universidade Federal do Espírito Santo - UFES; 2007.

21. Rondon MU, Forjaz CL, Nunes N, Amaral SL, Barretto AC, Negrão CE. Comparação entre a prescrição de intensidade de treinamento físico baseado na avaliação ergométrica convencional e na ergoespirométrica. Arq Bras Cardiol. 1998;70(3):159-66

22. American College of Sports Medicine. Diretrizes do ACSM para os testes de esforço e sua prescrição 5nd. ed. Rio de Janeiro: Guanabara Koogan; 2003.

23. Sousa MSC, Lima ACL, Silva JA, Silva GCC, Pontes LM, Lira FAS. Frequência cardiaca e pressão arteria em diferentes cargas no ergometro de Cirilo em mulheres ativas. Fit Perfor J. 2007;6(3):156-61.

24. Ceriani RB, Pontes LM, Sousa MSC. Consumo máximo de oxigênio em ergômetro banco: um estudo longitudinal em alunos do Núcleo Preparatório de Oficiais da Reserva. Fit Perf J. 2008;7(2):76-80

25. Chatterjee S, Chatterjee P, Bandyopadhyay A. Validity of Queen's College Step Test for estimation of maximum oxygen uptake in female students. Indian J Med Res. 2005;121(1):32-5.

26. American College of Sports Medicine.Guidelines for Exercise Testing and Prescription. 7nd. ed. Baltimore: Lippincott, Williams \& Wilkins; 2005

27. Shoenfeld Y, Kereen G, Birnfeld CH, Sohar E. Age, weight and heart rate at rest as predictors of aerobic fitness. J Sports Med Phys Fitness. 1981;21(4):377-82

28. Väinämö K, Nissilä S, Mäkikallio T, Tulppo M, Röning J. Artificial neural networks for aerobic fitness approximation. In: Proceedings of the International Neural Network ICNN. Washington, DC, 1996. 